\title{
JACQUES AGARD
}

\section{Treize ans de recherche opérationnelle appliquée à une entreprise}

Revue française d'automatique, d'informatique et de recherche opérationnelle. Recherche opérationnelle, tome 6, nº V1 (1972), p. 3-12.

<http://www.numdam.org/item?id=RO_1972_6_1_3_0>

() AFCET, 1972, tous droits réservés.

L'accès aux archives de la revue « Revue française d'automatique, d'informatique et de recherche opérationnelle. Recherche opérationnelle » implique l'accord avec les conditions générales d'utilisation (http://www.numdam.org/ legal.php). Toute utilisation commerciale ou impression systématique est constitutive d'une infraction pénale. Toute copie ou impression de ce fichier doit contenir la présente mention de copyright.

\section{Numdam}

Article numérisé dans le cadre du programme

Numérisation de documents anciens mathématiques

http://www.numdam.org/ 
R.A.I.R.O.

(6e année, $\mathrm{N}^{\circ} \mathrm{V}-1,1972$, p. 3-12)

\title{
TREIZE ANS DE REGHERGHE OPERATIONNELLE APPLIQUEE A UNE ENTREPRISE
}

\author{
par Jacques AGARD ( $\left.{ }^{1}\right)$
}

\begin{abstract}
Résumé. - L'auteur présente un bilan de fonctionnement d'une équipe; rattachement, moyens de calcul, membres de l'équipe, répartition des études et style du travail, méthodes les plus usitées. Les difficultés d'obtention et de choix des études, les réactions des clients de l'entreprise, l'efficacité des travaux et les conditions de succès sont ensuite analysées. Quelques problèmes d'actualité et des raisons de succès de la R.O. sont présentés pour finir.
\end{abstract}

Il y a deux ans, la Revue de Recherche Opérationnelle publiait un article "sur l'état de la Recherche Opérationnelle en France » $\left({ }^{2}\right)$. On y recensait les divers aspects de sa diffusion, et on constatait qu'elle intervenait «dans une vingtaine de sociétés de conseil. et une trentaine d'entreprises ». Cependant, si l'on ne prenait en compte que les groupes de R.O. où une dizaine de personnes au moins travaillent à temps complet, il est probable que les chiffres précédents seraient divisés par trois.

Pourtant, les techniques et l'état d'esprit R.O. se sont largement répandus, ne serait-ce que par la formation dispensée à présent dans l'enseignement supérieur, et grâce à l'informatique de gestion qui reprend à son compte de nombreux modèles de R.O.

Il nous a donc semblé intéressant de faire le point sur le groupe R.O. d'une entreprise, au terme de treize ans d'activité. Les constatations faites sur ce cas particulier sont seulement un témoignage et nous ne prétendons pas en tirer des recommandations. La transmission du savoir faire est difficile, de même que l'introspection sur les raisons des succès ou des échecs. Enfin, des modalités d'action localement efficaces peuvent dépendre de la structure et du contexte d'une entreprise, ainsi que de la personnalité des hommes qui les appliquent ou les subissent.

(1) Adjoint du Directeur du Programme et du Développement d'Air France, ancien Chef du Département de Recherche Opérationnelle d'Air France.

(2) R.I.R.O., 3 $3^{\mathrm{e}}$ année, no V-2, 1969, p. 3-11.

Revue Française d'Automatique, Informatique et Recherche Opérationnelle no V-1, 1972. 
Après ces précautions oratoires, est-il encore utile de conter une expérience particulière? Il nous semble que oui,car s'il n'existe pas de recettes universelles. il est des remarques qui peuvent confirmer nos sentiments, ou au contraire nous faire réfléchir sur le bien fondé de telle intuition : si cet article pouvait susciter une correspondance, des réflexions complémentaires, voire une polémique entre spécialistes, il aurait atteint son but. Signalons en passant qu'il y a quelques années, un groupe de travail de la section R,O, de l'AFCET s'était penché sur l'« efficacité de la R.O. ». Ne serait-il pas opportun de le réactiver, et trouverons-nous un animateur?

\section{L'historique d'un groupe R.O.}

Le groupe de R.O. d'Air France a vu le jour fin 1958. Créé par le Département Organisation de la Compagnie, il était constitué de 4 personnes : un spécialiste engagé à l'extérieur et trois ingénieurs de la maison. Durant les 8 ou 9 premières années, le groupe est resté au voisinage de cet effectif avec un appoint de 4 à 5 programmeurs scientifiques travaillant pour son compte à l'Informatique. Ce n'est que dans les quatre dernières années que l'importance du groupe a réellement augmenté pour atteindre une douzaine de chercheurs, un assistant et une secrétaire. Il existe toujours un appoint de 8 à 10 programmeurs travaillant sur des modèles d'origine R.O., et il faut citer l'apport croissant de stagiaires venant du Centre Interarmées de Recherche Opérationnelle, de l'Université ou de grandes Écoles qui collaborent très efficacement, bien que bénévolement, à des études concrètes.

Depuis 1967, le groupe a été transformé en Département, dans le sein de la Direction de l'Informatique et des Installations Fixes, rattachement qui le laisse, comme au Département Organisation, dans une position de neutralité hiérarchique et fonctionnelle souhaitable vis à vis des autres services de la Compagnie.

Enfin, il faut citer la cheville ouvrière du groupe : le terminal ordinateur en time sharing. Loué en 1968, il est devenu l'outil indispensable de la plupart de nos études et les jeunes cadres du groupe qui connaissent tous la programmation à leur arrivée à Air France en usent comme leurs anciens jouaient de la règle à calculs. La conception, la mise au point et l'expérimentation des modèles dans des délais assez rapides pour satisfaire nos clients dépendent fondamentalement de ce terminal.

\section{La constitution de l'équipe}

Depuis le début, la composition du groupe a peu varié; c'est un dosage d'universitaires scientifiques et d'ingénieurs de grandes écoles, ayant presque tous eu un premier frottement avec la R.O. par leur formation, des stages, un ou deux ans d'expérience, ou leur service militaire dans une unité R.O. A l'exception des premières années où l'un des membres du groupe était un 
ingénieur Air France d'une cinquantaine d'années, l'âge des cadres R.O. oscille entre 22 et 40 ans, la moyenne étant autour de 30 ans.

Les membres du groupe comprennent cependant des anciens et des nouveaux; les anciens viennent parfois d'autres services d'Air France, à moins qu'ils aient acquis leur expérience dans le groupe de R.O.; ils aident ainsi les nouveaux à trouver les bons correspondants pour chaque étude, à mieux comprendre le contexte de l'entreprise, et à profiter de l'expérience R.O. acquise sur d'autres champs de bataille.

La sélection des membres du groupe est faite à partir des candidatures ou en fonction des bons résultats d'un stage précédent. Si le groupe présente une cohésion remarquable et travaille dans une ambiance efficace et sympathique, il convient humblement de noter que nos méthodes de recrutement restent bien empiriques. Les chercheurs opérationnels ne devraient-ils pas réfléchir aux méthodes de sélection et d'adaptation des hommes aux postes, et rechercher des méthodes de validation en se prenant pour cobayes? Nous y pensons, mais le champ d'expériences du personnel R.O. est peut-être réduit pour des études statistiques sérieuses!

\section{La répartition du travail}

Chaque membre du groupe est chargé, seul ou en binome, de mener simultanément deux ou trois études dont la durée et l'importance sont variables. L'habitude croissante du travail en groupe dans les jeunes générations et la bonne entente de l'équipe R.O. multiplient heureusement les consultations et les conseils. Pour cela, chaque membre du groupe est spécialisé plus particulièrement sur quelques domaines d'applications et surtout sur quelques techniques dont il approfondit l'étude et rassemble la documentation. Il sert ainsi d'expert pour ses collègues utilisant cette technique. Le temps consacré aux compléments de formation, à la lecture de livres théoriques ou à la recherche est cependant trop réduit à notre gré, environ $15 \%$ à $20 \%$.

\section{Le fonctionnement du groupe}

Pour nous contraindre à ne pas perdre le contact de la théorie, nous demandons de temps à autre à chaque membre du groupe de présenter au cours de nos reunions de groupe bimensuelles une méthode intéressante qu'il a approfondie.

Lors de ces réunions de groupe sont exposés tour à tour les résultats et les méthodes de toutes les études en cours. La discussion peut apporter de nouvelles idées, mais l'exposé remplit surtout un double but : augmenter le nombre d'études concrètes dont chaque membre a connaissance et pourra s'inspirer et plus encore, habituer chaque chercheur à exposer clairement ses idées, et à répondre par avance aux critiques que lui feront plus tard ses clients.

$n^{\circ}$ V-1, 1972. 
Bien entendu, les membres du groupe ne sont pas seuls pour résoudre un problème.

Dans toute la mesure du possible, nous essayons d'avoir un groupe de travail incluant, au moins à temps partiel, nos correspondants d'autres services concernés par l'étude. C'est la meilleure façon de les intéresser, d'obtenir de bonnes informations, de les convaincre de la valeur des modèles et de les associer aux conclusions en vue de leur application.

Les étapes des études - position du problème, recensement des données et contraintes, modélisation, le plus souvent programmation, tests, expérimentation et validation de la méthode - sont accompagnées de rapports écrits, souvent sous la cosignature du service concerné et de la R.O. Ces rapports permettent des mises au point souvent utiles pour éviter des imprécisions ou des omissions, et pour informer les divers partenaires. Les conclusions écrites ou les modèles définitifs sont ensuite transmis aux décideurs, accompagnés de commentaires oraux. Les conclusions passent évidemment encore mieux si elles sont défendues par un membre du groupe de travail émanant du service auquel elles sont adressées.

En complément des études et des lectures, certains membres du groupe participent à des Symposiums, à des réunions de travail avec les groupes de R.O. d'autres Compagnies de transport aérien ou d'entreprises françaises. Ils donnent aussi quelques heures de cours ou de travaux pratiques dans l'enseignement supérieur pour approfondir la théorie et prendre l'habitude d'exposer un sujet.

Nous participons chaque année au Symposium réunissant les chercheurs opérationnels de toutes les Compagnies aériennes. Le brassage durant 5 jours de 60 chercheurs d'une vingtaine de Compagnies permet de mesurer les progrès des modèles R.O. dans l'industrie et de pêcher des idées originales.

\section{Les méthodes de R.O. utilisées}

Au cours de son existence, le groupe de R.O. d'Air France a utilisé la plupart des techniques de recherche opérationnelle, à l'exclusion de la théoriedes jeux.

Néanmoins, en fonction des connaissances des membres du groupe à cette époque ou de la nature des problèmes traités à ce moment là, il y a eu au fil du temps des méthodes plus usitées que d'autres. C'est ainsi que durant les premières années, la théorie des queues a été très souvent utilisée. Mais l'organisation des circuits de service et d'attente sort des hypothèses simplificatrices de la théorie, et les événements (services à fournir) ne sont pas répartis à la Poisson, mais sont liés aux horaires programmés de départ et d'arrivée des avions; nous avons donc recouru aux simulations pour résoudre nos problèmes de services avec arrivées quasi aléatoires. 
De nombreuses simulations d'emploi des flottes ou des services en escale ont ainsi été réalisées.

En ce domaine, les progrès accomplis dans la technique des calculateurs ont permis d'améliorer grandement nos possibilités en rendant les simulations plus réalistes, plus faciles à mettre en œuvre, et en réduisant leurs délais de mise au point. La balance a oscillé entre les simulations d'une réalité simplifiée et des mécanismes très détaillés et très complexes. L'efficacité est évidemment entre les deux : les modèles trop lourds n'étant plus compris ni dominés par leurs utilisateurs, et étant difficiles à adapter à l'évolution des systèmes.

Une autre méthode très usitée a été la méthode hongroise d'affectation. La mise au point par le groupe d'un algorithme efficace nous a incités à introduire cette méthode dans de nombreux cas d'affectation d'individus à des tâches, d'avions à des rotations ou à des postes de stationnement, de réemplois accrochant des opérations achevées à des opérations débutantes...

Le premier emploi de la programmation linéaire a été assez tardif, en affectant optimalement les divers types d'avions aux routes. Mais le retard a été vite rattrapé, et actuellement nous utilisons très largement les PL continus dans de nombreux problèmes. Très souvent, il est indispensable d'obtenir des solutions entières. On y parvient assez souvent lorsque les coefficients des variables et les second membres sont entiers. On se ramène donc à ce cas dans la mesure du possible. Lorsque certaines variables-solutions ne sont pas entières, nous utilisons des procédures heuristiques ou combinatoires pour arrondir la solution du P.L.

Pour résoudre les difficultés théoriques majeures, nous consultons nos collègues ou nous examinons la bibliographie; nos contacts avec l'Université et d'autres chercheurs nous mettent au fait des techniques utilisables et nous disposons de l'aide de conseillers scientifiques sur les difficultés théoriques. Nous n'avons donc pas recours à des consultants, bien que des consultants soient parfois appelés en renfort par d'autres services de la Compagnie sur des problèmes d'informatique et d'engineering.

\section{Le choix des études}

Lors des débuts du groupe d'Air France, il a été nécessaire de rechercher des sujets, et de proposer des études. Dans de nombreux cas, nous avons poursuivi des recherches sur des sujets auxquels les principaux responsables étaient médiocrement intéressés. Plus tard, des clients qui avaient apprécié notre aide nous ont sollicités pour de nouveaux problèmes. Le préjugé favorable de la Direction Générale et le côté réaliste et applicable de nos études ont peu à peu convaincu les services de notre sérieux, de sorte que les études demandées spontanément augmentent.

La sélection des problèmes attaqués par un groupe de R.O. se pose alors et est encore mal résolue. Cela rappelle la recherche minière à ses débuts. Au $n^{\circ}$ V-1, 1972. 
hasard des promenades d'un géologue et au flair d'un spéculateur, les filons les plus apparents étaient exploités, sans être toujours les plus rentables. L'ère scientifique est commencée depuis peu.

Dans ses débuts. un groupe de R.O. accepte le tout venant, et doit même proposer des études. En ce cas, il vaut mieux à notre avis aborder des domaines secondaires où les conclusions seront utiles, avec des modèles compréhensibles. Les erreurs éventuelles y seront sans conséquences graves. Après une période de rodage, le groupe pourra aborder des problèmes fondamentaux.

Lorsque le nombre des études augmente, il faut planifier leur mise en œuvre. Nous avons ainsi un plan quinquennal de nos travaux dans lequel les développements de grands problèmes sont prévus. Mais dans le cadre de l'année en cours se présente un tiers de travaux non programmés demandés en urgence par d'autres services. C'est dire que la planification des études à long terme est forcément incomplète.

En fait, il serait nécessaire que la Direction Générale fasse, une ou deux fois par an, un point des travaux et donne un ordre de priorité. Sinon, il faut se résigner à un choix un peu aléatoire où les sujets faciles ou accessibles prennent le pas sur des matières où l'aide de la R.O. serait autrement efficace.

Actuellement, le coût des études n'est pas débité aux services, bien qu'il soit pris en compte pour arrêter la liste d'études dont découlent les moyens et les budgets de la R.O.

\section{Les clients de la R.O.}

Depuis sa création, le groupe a travaillé pour toutes les directions d'Air France, ce qui lui donne collectivement une excellente connaissance des problèmes d'une Compagnie aérienne. Parmi les clients, de la R.O., on peut établir plusieurs classes :

- les clients spontanés ne sont pas encore très nombreux, ne serait-ce que parce qu'il leur est difficile de décider si une étude relève de la R.O.

Parmi ces clients, nous trouvons des services qui ont déjà eu recours à notre aide, ce qui est un bon indice de notre succès.

Il faut même parfois ramener aux réalités certains enthousiastes qui espérent trop et trop vite de nos techniques.

- les clients coopératifs sont d'accord pour participer à une étude proposée par nous, et à ses développements.

- les clients passifs fournissent les informations demandées, mais n'ont pas le temps ou le goût de s'intégrer au mouvement.

- les sceptiques et les hostiles ne croient pas aux techniques modernes, craignent la critique possible, estiment ne pouvoir comprendre ou n'ont pas envie d'adapter leurs méthodes. 
Pour convaincre ces derniers de l'intérêt de la R.O. et pour exposer à tous les autres ses possibilités, il faut informer les cadres de l'entreprise.

Dans ce but, nous avons autrefois diffusé des brochures sur la R.O., ses méthodes et ses applications, Nous avons organisé quelques conférences par petits groupes sur des sujets spécifiques.

Pour les cadres supérieurs ventilés en groupes de 20 à 30 personnes, une journée plusieurs fois répétée sur la R.O. a fait partie d'un cycle de conférences organisé par la Direction du personnel. Si les participants nous ont dit être satisfaits de ces conférences, nous devons ajouter qu'aucune retombée d'étude n'en est résulté. On peut cependant espérer que les participants en ont retenu des idées pour aménager leur gestion. Cependant, l'impact de ces efforts de formation, comme celui des contacts de travail entre membres du groupe R.O. et d'autres services, a sans doute contribué à créer un climat satisfaisant : les chercheurs opérationnels ont dans l'ensemble bonne presse dans l'entreprise et interviennent toujours dans un environnement amical.

\section{L'efficacité des études}

Au terme d'une étude, il serait souhaitable de pouvoir établir un bilan pour contrôler son efficacité par rapport aux espoirs émis lors du diagnostic préalable à son lancement. Ce bilan est malheureusement très délicat à établir, mais on peut nuancer comme suit l'utilité d'une étude de R.O. :

- l'efficacité nulle est la plus simple : elle correspond à une étude dont on peut démontrer les erreurs d'hypothèses ou de méthode a posteriori. On y apprend à ne plus commettre certaines erreurs;

- l'étude non avenue est mise dans un tiroir et n'attire aucune remarque : on ne saura jamais s'il faut mettre en cause sa qualité ou la valeur du décideur.

C'est un cas assez fréquent dont le chercheur opérationnel ne peut tirer aucune leçon.

- l'étude est prématurée: les esprits ne sont pas prêts à appliquer certaines méthodes ou le problème n'est pas encore assez complexe pour dépasser l'intuition du responsable. Quelques années plus tard, le même modèle est appliqué avec succès.

- l'étude est appliquée : par exemple elle donne lieu à un programme informatique employé régulièrement pour calculer des moyens et prendre des décisions : c'est l'emploi normal de nombreux modèles pour lesquels le chercheur n'a pas perdu son temps.

- l'étude est rentable : l'utilisation régulière d'un modèle ne prouve rien sur sa valeur. Il faut encore prouver sa rentabilité ou sa nécessité. Certaines méthodes de R.O. peuvent conduire, du fait de l'automatisation, à un accroissement de coût par rapport au passé, mais on peut prouver que l'extrapola$n^{\circ}$ V-1, 1972. 
tion des procédés empiriques n'était plus possible sans conduire à une grave détérioration de qualité (par exemple de bonnes solutions artisanales font faillite lorsque la charge croît et conduit à des systèmes industriels).

La mesure de l'efficacité est toujours délicate et contestable : une étude de R.O. peut entraîner des gains économiques, des améliorations de qualité (réduction d'attentes, de retards...), ou accroître la sécurité du décideur en mesurant l'écart d'autres solutions et en fournissant des fourchettes sur les conséquences de fluctuations aléatoires. Mais, toutes ces comparaisons doivent être faites par rapport à la reconduction de méthodes empiriques qui auraient peut-être évolué!

- l'étude accompagne une décision nécessaire : pour toute décision importante, il faut bien envisager diverses stratégies, explorer des options, effectuer des chiffrages. La R.O. fournit un cadre rationnel à ces analyses. La modélisation pratiquée accroît peut-être les délais et le coût, mais en son absence, l'intuition des décideurs aurait-elle conduit au bon choix?

- la R.O. modifie les points de vue : même lorsque les recommandations de la R.O. ne sont pas appliquées dans des décisions importantes de gestion, leur influence joue indirectement. Les responsables ou leur entourage ont en effet mieux pris conscience des possibilités du calcul économique, des incidences des facteurs aléatoires, de l'intérêt d'étudier des variantes. Cette évolution des esprits est inégale et peu mesurable : elle est d'autant plus rapide que la direction générale est favorable aux méthodes modernes de gestion et exige des solutions de rechange ou des fourchettes d'erreur.

Le développement de l'Informatique, en ouvrant de nouvelles possibilités de calcul et de traitement des données, pousse également à élaborer des modèles pour tirer un meilleur usage des calculateurs et pour décider en connaissance de cause.

\section{Les conditions du succès}

Après ces considérations un peu désabusées sur la mesure de l'efficacité de la R.O., peut-on parler du succès ou doit-on plutôt parler de la santé du groupe de R.O.? En tout cas. sa survie et sa bonne réputation à long terme nous paraissent dépendre de plusieurs conditions.

- la diplomatie et l'aménité sont indispensables. Un chercheur opérationnel doit être sociable, courtois et discret avec ses correspondants;

- l'aptitude au travail en groupe est essentielle, avec la sociabilité, l'acceptation de la critique, le sens de l'humour et l'esprit de camaraderie;

- le goût du concret doit conduire à traiter les problèmes dans le contexte où ils se posent, et à renoncer parfois à tel développement théorique séduisant mais un peu superflu, dont l'utilisateur aura le plus grand mal à comprendre l'intérêt; 
- la présentation des résultats : par une compensation de la rigueur apportée lors de la modélisation et de la programmation, ou plutôt par inexpérience, l'attention du chercheur se relâche lors de la rédaction et de la présentation des résultats. Un rapport mal présenté pour gagner quelques jours risque de gâcher le succès d'une étude qui a nécessité des mois d'effort et d'imagination;

- la participation des clients doit être obtenue en veillant à ce que l'essentiel du modèle soit compris, en associant les partenaires aux rapports, aux discussions, aux conclusions, en leur laissant l'emploi des modèles après leur achèvement.

On peut dire que le succès du groupe est atteint lorsque d'autres directions cherchent à faire muter ses membres dans leurs services. Ce sera une nouvelle aide à la diffusion de la R.O., à condition que cela ne soit pas pour créer des groupes R.O. concurrents au sein de l'entreprise. Ce n'est pas là une crainte de la compétition éprouvée par le groupe central de R.O., mais la certitude que des sous-groupes travailleront au contraire sur des domaines réservés avec une efficacité aussi réduite que la taille du sous-groupe.

\section{Les sujets porteurs de succès}

Existe-t-il actuellement des sujets d'étude rentables pour un groupe R.O.? Encore une fois, les bons sujets d'étude dépendent du secteur et des particularités de l'entreprise, mais on peut cependant signaler quelques domaines en développement, pour ne pas citer à nouveau les sujets classiques exposés dans la littérature :

- les problèmes du personnel, gestion et embauche à long terme, sélection et affectation, adaptation des hommes aux postes ont actuellement une vogue croissante;

- les modèles de gestion : les modèles d'entreprise, la gestion de trésorerie, les choix d'investissements, la productivité globale des facteurs offrent des développements nouveaux aux études économiques classiques;

- les modèles informatisés : 1'Informatique, pour sortir de la phase de stockage et de transcription de données, fait de plus en plus appel aux techniques R.O. pour présenter des résultats élaborés (par exemple automatisation de plannings de stewards et hôtesses, calcul de charges d'escale, calcul des rotations d'avions ou d'équipages, modèles de stock...);

- le marketing offre un domaine encore peu exploré (prévision du marché, publicité, comportement du consommateur);

- l'analyse des données : les progrès accomplis en analyse factorielle, composantes principales, méthodes de segmentation... devraient nous permettre dans les prochaines années de tirer le meilleur parti des informations aussi nombreuses que mal choisies en raison de notre méconnaissance des phénomènes lors du recueil des données.

$\mathrm{n}^{0} \mathrm{~V}-1,1972$. 


\section{Un renouveau de la R.O.}

Après les espérances parfois exagérées de certains dans les années 1950, la recherche opérationnelle a un peu marqué le pas et de nombreux groupes ou sociétés conseils, lancés avec la mode, ont disparu ou stagné : les méthodes étaient mal rodées, les calculateurs inadaptés aux modèles. En outre, les dirigeants d'entreprise étaient le plus souvent méfiants envers des techniques qu'ils ne connaissaient pas par manque de formation scientifique, ou qu'ils étaient dépités de ne pas comprendre malgré leur bagage scientifique un peu ancien. Il faut aussi incriminer la maladresse de certains chercheurs trop théoriciens et leur manque d'habitude du travail en groupe.

Depuis, les calculateurs et les softwares sont devenus compatibles avec des modèles complexes traitant des milliers de variables ou des millions d'opérations. Les méthodes se sont décantées et on a appris à simplifier lorsque c'était possible. Enfin, les chercheurs, issus depuis peu de l'enseignement supérieur, ont davantage l'habitude du travail en groupe.

Mais surtout, les efforts des pionniers et l'enseignement des méthodes scientifiques de gestion diffusé un peu partout ont habitué les correspondants au langage et aux méthodes de la R.O. Les décideurs trouvent donc dans leurs services des jeunes cadres servant de relai et faisant passer le message au sommet. Il convient également de signaler la vogue croissante des méthodes de "Rationalisation des Choix Budgétaires » qui gagne les administrations et les grandes entreprises. Les patrons retrouvent là à leur niveau l'attitude d'esprit du chercheur opérationnel. Pour simuler diverses politiques, réaliser des études quialité-coût, apprécier la sensibilité de divers programmes aux aléas, il est indispensable que la RCB (ou le PPBS) recoure à la R.O.

Le passage d'un jeune cadre dans un service de recherche opérationnelle ou d'informatique n'est donc plus une tare ni une spécialisation définitive, mais une preuve de son ouverture d'esprit, et une formation complémentaire.

La recherche opérationnelle nous semble ainsi une occasion d'avoir une vue globale de l'entreprise avec le recul souhaitable et une formation à la gestion, préalables à des carrières ouvertes sur tous les domaines de l'entreprise. 